\title{
Charged perfect fluid disks as sources of Taub-NUT-type spacetimes
}

\author{
Gonzalo García-Reyes: and Guillermo A. Gonzáled \\ Escuela de Física, Universidad Industrial de Santander, A. A. 678, Bucaramanga, Colombia
}

\begin{abstract}
The interpretation of a family of electrovacuum stationary Taub-NUT-type fields in terms of finite charged perfect fluid disks is presented. The interpretation is mades by means of an "inverse problem" approach used to obtain disk sources of known solutions of the Einstein or EinsteinMaxwell equations. The diagonalization of the energy-momentum tensor of the disks is facilitated in this case by the fact that it can be written as an upper right triangular matrix. We find that the inclusion of electromagnetic fields changes significatively the different material properties of the disks and so we can obtain, for some values of the parameters, finite charged perfect fluid disks that are in agreement with all the energy conditions.
\end{abstract}

PACS numbers: 04.20.-q, 04.20.Jb, 04.40.Nr

\section{INTRODUCTION}

Several methods are known to exactly solve the Einstein and Einstein-Maxwell field equations, or to generate new exact solutions from simple known solutions [1]. However, the above mentioned methods in general lead to solutions without a clear physical interpretation or to solutions that depend of many parameters without a clear physical meaning. Accordingly, it is of importance to have some appropriate procedures to obtain physical interpretations of these exact solutions. So, in the past years such procedures have been developed for static and stationary axially symmetric solutions in terms of thin and, more recently, thick disk models.

Stationary or static axially symmetric exact solutions of Einstein equations describing relativistic thin disks are of great astrophysical importance since they can be used as models of certain stars, galaxies and accretion disks. These were first studied by Bonnor and Sackfield [2], obtaining pressureless static disks, and then by Morgan and Morgan, obtaining static disks with and without radial pressure [3, 4]. In connection with gravitational collapse, disks were first studied by Chamorro, Gregory, and Stewart [5]. Disks with radial tension have been also studied [6]. Recently, more realistic models of thin disks and thin disks with halos made of perfect fluids were considered in [7].

Several classes of exact solutions of the Einstein field equations corresponding to static and stationary thin disks have been obtained by different authors $[8,9,10$, $11,12,13,14,15,16,17,18$, with or without radial pressure. Except for the pressureless disks, generally the other disks have as source matter with azimuthal pressure (tension) different from the radial pressure (tension). However, in some cases these disks can be interpreted as the superposition of two counterrotating perfect fluids. A detailed study of the counterrotating model for the case of static thin disks is presented in [19].

\footnotetext{
*Email address: ggr1970@yahoo.com

${ }^{\dagger}$ Email address: guillego@uis.edu.co
}

Disk sources for stationary axially symmetric spacetimes with magnetic fields are also of astrophysical importance mainly in the study of neutron stars, white dwarfs and galaxy formation. Although disks with electric fields do not have clear astrophysical importance, their study may be of interest in the context of exact solutions. Thin disks have been discussed as sources for Kerr-Newman fields [20], magnetostatic axisymmetric fields 21], conformastationary metrics 22], and electrovacuum static counterrotating disks [23].

In all the above cases, the disks are obtained by an "inverse problem" approach, called by Synge the " $g$-method" 24]. The method works as follows: a solution of the vacuum Einstein equations is taken, such that there is a discontinuity in the derivatives of the metric tensor on the plane of the disk, and the energy-momentum tensor is obtained from the Einstein equations. The physical properties of the matter distribution are then studied by an analysis of the surface energy-momentum tensor so obtained. Very recently the above procedure has been generalized in order to obtain thick disk models [25] and also non-axisymmetric planar distributions of charged dust [26]. As we can see, by this "inverse problem" approach we can do physical interpretations of many known solutions of the Einstein and Einstein-Maxwell vacuum equations, in the sense that the thin disks can act as exact sources for the space-time metrics given by the vacuum solutions.

The purpose of this paper is the interpretation of a family of electrovacuum stationary Taub-NUT-type spacetimes in terms of finite charged perfect fluid disks. The paper is structured as follows. We first present, in Sec. II, the family of stationary Taub-NUT-type solutions of the Einstein-Maxwell equations that we will consider. Then, in Sec. III, we present a summary of the procedure to construct models of disks with nonzero radial pressure. We obtain expressions for the surface energy-momentum tensor and the surface current density of the disks. In particular we find explicit expressions for the surface energy density, the pressure and the surface charge electric density. Finally, the disks are interpreted in terms of a charged perfect fluid. In Sec. IV we analyze the physical properties of the disks and we show that, for 
some values of the parameters, the disks are in agreement with all the energy conditions. We also study the motion of the disks and their stability against radial perturbations is analyzed. Finally, in Sec. V, we summarize our main results.

\section{EINSTEIN-MAXWELL EQUATIONS AND TAUB-NUT-TYPE FIELDS}

The line element of a stationary axisymmetric field can be written in quasi-cylindrical coordinates $(\mathrm{t}, \varphi, r, z)$ in the form

$d s^{2}=-e^{2 \Psi}(d t+\mathcal{W} d \varphi)^{2}+e^{-2 \Psi}\left[R^{2} d \varphi^{2}+e^{2 \Lambda}\left(d r^{2}+d z^{2}\right)\right]$,

where $R, \Psi, \mathcal{W}$, and $\Lambda$ are functions of $r$ and $z$ only. The vacuum Einstein-Maxwell equations, in geometrized units in which $8 \pi G=c=\mu_{0}=\varepsilon_{0}=1$, are given by

$$
\begin{aligned}
& G_{a b}=T_{a b}, \\
& F_{; b}^{a b}=0,
\end{aligned}
$$

with

$$
\begin{aligned}
T_{a b} & =F_{a c} F_{b}{ }^{c}-\frac{1}{4} g_{a b} F_{c d} F^{c d}, \\
F_{a b} & =A_{b, a}-A_{a, b} .
\end{aligned}
$$

For the metric (1), the Einstein-Maxwell equations in vacuum imply that $R$ satisfies the Laplace's equation

$$
R_{, r r}+R_{, z z}=0
$$

so that the function $R$ can be considered as the real part of an analytical function $F(\nu)=R(r, z)+i Z(r, z)$, where $\nu=r+i z$. Thus the function $F(\nu)$ defines a conformal transformation

$$
\begin{aligned}
& r \rightarrow R(r, z), \\
& z \rightarrow Z(r, z),
\end{aligned}
$$

in such a way that the metric (1) takes the usual WeylLewis-Papapetrou form

$d s^{2}=-e^{2 \tilde{\Psi}}(d t+\tilde{\mathcal{W}} d \varphi)^{2}+e^{-2 \tilde{\Psi}}\left[R^{2} d \varphi^{2}+e^{2 \tilde{\Lambda}}\left(d R^{2}+d Z^{2}\right)\right]$

where $R$ and $Z$ are the Weyl's canonical coordinates. In this coordinates, the field equations (2) are equivalent to the usual complex Ernst equations [27]

$$
\begin{aligned}
& f \Delta \mathcal{E}=\left(\nabla \mathcal{E}+2 \Phi^{*} \nabla \Phi\right) \cdot \nabla \mathcal{E}, \\
& f \Delta \Phi=\left(\nabla \mathcal{E}+2 \Phi^{*} \nabla \Phi\right) \cdot \nabla \Phi,
\end{aligned}
$$

where $\Delta$ and $\nabla$ are the standard differential operators in cylindrical coordinates and $f=e^{2 \tilde{\Psi}}$. The metric functions and the electromagnetic potentials are obtained from the relations

$$
\begin{aligned}
f & =\operatorname{Re} \mathcal{E}+\Phi \Phi^{*}, \\
4 f^{2} \tilde{\Lambda}_{, \zeta} & =\sqrt{2} R\left(\mathcal{E}_{, \zeta}+2 \Phi^{*} \Phi_{, \zeta}\right)\left(\mathcal{E}_{, \zeta}^{*}+2 \Phi \Phi_{, \zeta}^{*}\right)-4 f \Phi_{, \zeta}\left(\Phi_{, \zeta},{ }_{,}\right) \\
f^{2} \tilde{\mathcal{W}}_{, \zeta} & =R\left[i(\operatorname{Im} \mathcal{E})_{, \zeta}+\Phi^{*} \Phi_{, \zeta}-\Phi \Phi_{, \zeta}^{*}\right], \\
\tilde{A}_{t} & =\sqrt{2} \operatorname{Re} \Phi, \\
f \tilde{A}_{\varphi, \zeta} & =\sqrt{2}\left[i R(\operatorname{Im} \Phi)_{, \zeta}+\tilde{\mathcal{W}} \tilde{A}_{t, \zeta}\right],
\end{aligned}
$$

where $\sqrt{2} \zeta=R+i Z$, so that $\sqrt{2} \partial_{, \zeta}=\partial_{, R}-i \partial_{, Z}$.

We will consider a simple stationary Taub-NUT-type solution to the above system of equations given by

$$
\begin{aligned}
\tilde{\Psi} & =\frac{1}{2} \ln \left[\frac{x^{2}-1}{x^{2}+2 a x+1+c^{2}}\right], \\
\tilde{\Lambda} & =\frac{1}{2} \ln \left[\frac{x^{2}-1}{x^{2}-y^{2}}\right], \\
\tilde{\mathcal{W}} & =2 k b y \\
\tilde{A}_{t} & =\frac{\sqrt{2} c}{\sqrt{1+c^{2}}}\left[\frac{1+c^{2}+a x}{x^{2}+2 a x+1+c^{2}}\right], \\
\tilde{A}_{\varphi} & =\frac{\sqrt{2} c k b y}{\sqrt{1+c^{2}}}\left[\frac{1+c^{2}-x^{2}}{x^{2}+2 a x+1+c^{2}}\right],
\end{aligned}
$$

where $a^{2}+b^{2}=1+c^{2}$, being $c$ the parameter that controls the electromagmetic field. The prolate spheroidal coordinates, $x$ and $y$, are related to the Weyl coordinates by

$$
R^{2}=k^{2}\left(x^{2}-1\right)\left(1-y^{2}\right), \quad Z=k x y,
$$

where $1 \leq x \leq \infty$ and $0 \leq y \leq 1$. This solution can be generated, in these coordinates, using the well-known complex potential formalism proposed by Ernst [27] from the Taub-NUT vacuum solution [1]. Note that when $c=0$ this solution reduces to the Taub-NUT vacuum solution.

Once the above solution is known, we can obtain a solution of the field equations (2) in the original coordinates 
by setting

$$
\begin{aligned}
R(r, z) & =\operatorname{Re} F(\nu) \\
\Psi(r, z) & =\tilde{\Psi}(R, Z) \\
\Lambda(r, z) & =\tilde{\Lambda}(R, Z)+\ln \left|F^{\prime}(\nu)\right| \\
\mathcal{W}(r, z) & =\tilde{\mathcal{W}}(R, Z), \\
A_{t}(r, z) & =\tilde{A}_{t}(R, Z), \\
A_{\varphi}(r, z) & =\tilde{A}_{\varphi}(R, Z),
\end{aligned}
$$

where $F^{\prime}=d F / d \nu$. Note also that the solution to the original Einstein-Maxwell equations (2) is not completely determined as we do not take any especific choice for $F(\nu)$. We will do this in the next section.

\section{A FAMILY OF FINITE CHARGED PERFECT FLUID DISKS}

In order to obtain a solution of (2) representing a thin disk at $z=0$, we assume that the components of the metric tensor and the electromagnetic potentials are continuous across the disk, but with first derivatives discontinuous on the plane $z=0$, with discontinuity functions

$$
\begin{aligned}
b_{a b} & =\left.g_{a b, z}\right|_{z=0^{+}}-\left.g_{a b, z}\right|_{z=0^{-}}=\left.2 g_{a b, z}\right|_{z=0^{+}}, \\
a_{a} & =\left.A_{a, z}\right|_{z=0^{+}}-\left.A_{a, z}\right|_{z=0^{-}}=\left.2 A_{a, z}\right|_{z=0^{+}} .
\end{aligned}
$$

Thus, the Einstein-Maxwell equations yield an energymomentum tensor $T_{a b}=T_{a b}^{\mathrm{elm}}+T_{a b}^{\mathrm{mat}}$, where $T_{a b}^{\mathrm{mat}}=$ $Q_{a b} \delta(z)$, and a current density $J_{a}=j_{a} \delta(z)=$ $-2 e^{2(\Psi-\Lambda)} A_{a, z} \delta(z)$, where $\delta(z)$ is the usual Dirac function with support on the disk. $T_{a b}^{\mathrm{elm}}$ is the electromagnetic tensor defined in Eq. (3a), $j_{a}$ is the current density on the plane $z=0$, and

$Q_{b}^{a}=\frac{1}{2}\left\{b^{a z} \delta_{b}^{z}-b^{z z} \delta_{b}^{a}+g^{a z} b_{b}^{z}-g^{z z} b_{b}^{a}+b_{c}^{c}\left(g^{z z} \delta_{b}^{a}-g^{a z} \delta_{b}^{z}\right)\right\}$

is the distributional energy-momentum tensor. The "true" surface energy-momentum tensor (SEMT) of the disk, $S_{a b}$, and the "true" surface current density, $j_{a}$, can be obtained through the relations

$$
\begin{aligned}
S_{a b} & =\int T_{a b}^{\text {mat }} d s_{n}=e^{\Lambda-\Psi} Q_{a b}, \\
j_{a} & =\int J_{a} d s_{n}=e^{\Lambda-\Psi} j_{a},
\end{aligned}
$$

where $d s_{n}=\sqrt{g_{z z}} d z$ is the "physical measure" of length in the direction normal to the disk.
For the metric (1), the nonzero components of $S_{a}^{b}$ are

$$
\begin{aligned}
S_{0}^{0} & =\frac{e^{\Psi-\Lambda}}{R^{2}}\left[2 R^{2}(\Lambda, z-2 \Psi, z)+2 R R, z-e^{4 \Psi} \mathcal{W} \mathcal{W},_{z}\right](13 \mathrm{a}) \\
S_{1}^{0} & =\frac{e^{\Psi-\Lambda}}{R^{2}}\left[2 R \mathcal{W}(R, z-2 R \Psi, z)-\left(R^{2}+\mathcal{W}^{2} e^{4 \Psi}\right) \mathcal{W}(133 \mathrm{~b})\right. \\
S_{0}^{1} & =\frac{e^{\Psi-\Lambda}}{R^{2}}\left[e^{4 \Psi} \mathcal{W},,_{z}\right] \\
S_{1}^{1} & =\frac{e^{\Psi-\Lambda}}{R^{2}}\left[2 R^{2} \Lambda,_{z}+e^{4 \Psi} \mathcal{W} \mathcal{W},_{z}\right] \\
S_{2}^{2} & =\frac{e^{\Psi-\Lambda}}{R^{2}}[2 R R, z]
\end{aligned}
$$

and the nonzero components of the surface current density $j_{a}$ are

$$
\begin{aligned}
& j_{t}=-2 e^{\Psi-\Lambda} A_{t, z}, \\
& j_{\varphi}=-2 e^{\Psi-\Lambda} A_{\varphi, z},
\end{aligned}
$$

where all the quantities are evaluated at $z=0^{+}$.

We now consider a solution with the above mentioned discontinuity properties by taking the Taub-NUT-type solution (9) with $F(\nu)$ given by

$$
F(\nu)=\nu+\alpha \sqrt{\nu^{2}-1}
$$

where $\alpha \geq 0$. This choice for $F(\nu)$ was firstly presented in reference [6] and leads to thin disks with nonzero radial pressure and of finite radius, located at $z=0,0 \leq r \leq 1$. In order to obtain disks with non-unit radius, we only need to make the transformation $r \rightarrow a r$, where $a$ is the radius of the disk.

With this choice for $F(\nu)$ the image of the disk by the conformal mapping (5) is the surface

$$
\alpha^{2} R^{2}+Z^{2}=\alpha^{2}
$$

so that the disks are mapped into spheroidal thin shells of matter and its exterior is mapped into the exterior of shells. We have three possible values for $\alpha$ : $\alpha=1$, a spherical shell, $\alpha>1$, a prolate spheroidal shell, and $0<\alpha<1$, an oblate spheroidal shell. Therefore, the coordinates naturally adapted to the symmetry of the shells are, respectively, spherical, prolate spheroidal and oblate spheroidal. By considering the above shells as sources, we then seek for exterior solutions of the field equations (7) and, using (11), we obtain the corresponding disk solutions in the original coordinates. Thus, for example, for the Taub-NUT-type metric (9), written in prolate spheroidal coordinates, we must choose $\alpha>1$ and the shell would be located at $x=\alpha / k>1$ and $y=\sqrt{1-r^{2}}$, with $k=\sqrt{\alpha^{2}-1}$.

Due to the fact that the metric function $\mathcal{W}(r, z)$ depends only of the prolate spheroidal coordinate $y$, and as 
a consequence of the behavior of the derivatives of $F(\nu)$, we can easily see that $\mathcal{W},_{z}=0$ at the disk and so we have $S_{0}^{1}=0$ and $S_{1}^{0}=\left(S_{0}^{0}-S_{1}^{1}\right) \mathcal{W}$. That is, the SEMT can be written as an upper right triangular matrix and so can be easily diagonalized in terms of an orthonormal tetrad $\mathrm{e}_{\hat{a}}^{b}=\left\{V^{b}, W^{b}, X^{b}, Y^{b}\right\}$, where

$$
\begin{aligned}
V^{a} & =e^{-\Psi}(1,0,0,0), \\
W^{a} & =\frac{e^{\Psi}}{R}(-\mathcal{W}, 1,0,0), \\
X^{a} & =e^{\Psi-\Lambda}(0,0,1,0), \\
Y^{a} & =e^{\Psi-\Lambda}(0,0,0,1) .
\end{aligned}
$$

Since the vectors $\mathrm{e}_{\hat{a}}^{b}$ are the eigenvectors of the SEMT, the timelike vector $V^{a}$ can be interpreted as the velocity vector of the disks and so the orthonormal tetrad is comoving. This is the orthonormal tetrad used by static observers who are at rest with respect to infinity, or "Locally Static Observers" (LSO) [22].

We obtain for the metric, the SEMT and the surface current density the expressions

$$
\begin{aligned}
g_{a b} & =-V_{a} V_{b}+W_{a} W_{b}+X_{a} X_{b}+Y_{a} Y_{b}, \\
S_{a b} & =\epsilon V_{a} V_{b}+p_{\varphi} W_{a} W_{b}+p_{r} X_{a} X_{b}, \\
j_{a} & =\sigma V_{a}+j W_{a},
\end{aligned}
$$

where

$$
\epsilon=-S_{0}^{0}, \quad p_{\varphi}=S_{1}^{1}, \quad p_{r}=S_{2}^{2},
$$

are, respectively, the surface energy density, the azimuthal pressure, and the radial pressure measured by this observer, and

$$
\sigma=-V^{0} j_{0}, \quad j=W^{0} j_{0}+W^{1} j_{1},
$$

are the electric charge density and the azimutal current density of the disk measured by this observer.

From the expressions (9) for the Taub-NUT-type solution, is easy to see that $p_{\varphi}=p_{r}=p$ and so the surface energy-momentum tensor can be cast in the perfect fluid form

$$
S_{a b}=(\epsilon+p) V_{a} V_{b}+p h_{a b}
$$

where $h_{a b}=g_{a b}-Y_{a} Y_{b}$ is the metric of the $z=0$ hipersurface,

$$
\begin{aligned}
& \epsilon=-\left[\frac{\bar{\alpha}+a}{2 k^{2}}\right]\left(\frac{p}{\bar{\alpha}}\right)^{3}, \\
& p=\frac{2 \bar{\alpha}}{\sqrt{\bar{\alpha}^{2}+2 a \bar{\alpha}+1+c^{2}}}
\end{aligned}
$$

where $\bar{\alpha}=\alpha / k>1$. From (22a) follows that the fluid has a barotropic equation of state. The effective Newtonian density, defined as $\varrho=\epsilon+2 p$, is

$$
\varrho=\left[\frac{a\left(\bar{\alpha}^{2}+1\right)+\left(2+c^{2}\right) \bar{\alpha}}{2}\right]\left(\frac{p}{\bar{\alpha}}\right)^{3} .
$$

The surface current density in the coordinates frame is given by

$$
\begin{aligned}
& j_{t}=c\left[\frac{a \bar{\alpha}^{2}+\left(1+c^{2}\right)(2 \bar{\alpha}+a)}{8 \sqrt{2} k^{2}\left(1+c^{2}\right)^{1 / 2}}\right]\left(\frac{p}{\bar{\alpha}}\right)^{5}, \\
& j_{\varphi}=2 k b\left(1-r^{2}\right)^{1 / 2} j_{t},
\end{aligned}
$$

whereas the surface electric charge density measured by the comoving observer is

$$
\sigma=-c\left[\frac{a \bar{\alpha}^{2}+\left(1+c^{2}\right)(2 \bar{\alpha}+a)}{4 \sqrt{2} k\left(1+c^{2}\right)^{1 / 2}}\right]\left(\frac{p}{\bar{\alpha}}\right)^{4}
$$

and the azimuthal current density measured by the comoving observer is equal to zero. Thus we have a family of finite charged perfect fluid disks with constant surface energy density and pressure given by (22), and constant surface electric charge density given by (25).

\section{THE PHYSICAL PROPERTIES OF THE DISKS}

We will now analyze the physical properties of the disks. In first instance we consider that $c=0$, so that $a^{2}+b^{2}=1$. In this case the electromagnetic potentials are equal to zero and so the disks are made of a neutral perfect fluid. Furthermore, is easy to see that with $|a| \leq 1$ the energy density is always a negative quantity and so the disks obtained when $c=0$ never satisfy the weak energy condition. So, in order to have disks in agreement with the weak energy condition, we need to consider solutions with $c \neq 0$. That is, disks with nonzero surface electric charge density.

From expression (22a) is easy to see that the energy density is positive if we take

$$
a<-\bar{\alpha}<-1
$$

Additionally, in order to have real positive expressions for the pressure $p$ and the effective Newtonian density $\varrho$, we must to impose the conditions

$$
\begin{aligned}
\bar{\alpha}^{2}+2 a \bar{\alpha}+1+c^{2} & >0, \\
a\left(\bar{\alpha}^{2}+1\right)+\left(2+c^{2}\right) \bar{\alpha} & \geq 0 .
\end{aligned}
$$

Thus, for example, if we take $c=3, a=-3$ and $\bar{\alpha}=2$, 
we have that

$$
\begin{aligned}
& \epsilon=3 \sqrt{2}, \\
& p=2 \sqrt{2}, \\
& \varrho=7 \sqrt{2}, \\
& \sigma=0.6 \sqrt{15},
\end{aligned}
$$

in agreement with the weak and strong energy conditions. Furthermore, $p<\epsilon$ and so the disk also satisfies the dominant energy condition.

In order to analyze the motion of the disks, we can see that the spatial components of the velocity vector $V^{a}$ are zero with respect to the coordinates and so the disks are "locally static". The motion of the disks can also be analyzed by considering an orthonormal frame different from the comoving tetrad. So, an orthonormal tetrad commonly used is the "Locally Nonrotating Frame" or "Zero Angular Momentum Observer" [29, 30]. The tangential velocity of the disks measured by this observer is given by

$$
v_{L N R F}=\frac{g_{11}(\Omega-\omega)}{\sqrt{g_{01}^{2}-g_{00} g_{11}}},
$$

where $\omega=-g_{01} / g_{11}$ and $\Omega=V^{1} / V^{0}$. We obtain

$$
v_{L N R F}=-\frac{b}{2 k}\left(\frac{p}{\bar{\alpha}}\right)^{2} \frac{\sqrt{1-r^{2}}}{r} .
$$

As we can see from the above expression, with respect to LNRF, the particles of the disks move with superluminal velocities for $r<r_{0}$, where

$$
r_{0}=\frac{b p^{2}}{\sqrt{b^{2} p^{4}+4 k^{2} \bar{\alpha}^{4}}} .
$$

For instance, with the values used in (28), we have that $r_{0}=\sqrt{12 / 13} \approx 0.96$, and so the disks will have subluminal velocities only in a narrow region near the edge. However, the above superluminal behavior of the velocity is indeed due to the motion of the LNRF and not to the motion of the disks. So, we can see that for this family of solutions the LNRF is not a well behaved observer in the sense that the frame itself presents superluminal velocities.

Another quantity related with the motion of the disk is the specific angular momentum of a particle rotating at a radius $r$, defined as $h=g_{\varphi a} V^{a}$. Thus we have

$$
h^{2}=\left(\frac{b p}{\bar{\alpha}}\right)^{2} \sqrt{1-r^{2}} .
$$

This quantity can be used to analyze the stability of the disks against radial perturbations. The condition of stability,

$$
\frac{d\left(h^{2}\right)}{d r}>0
$$

is an extension of Rayleigh criteria of stability of a fluid in rest in a gravitational field [28]. As we can see, the disks are not stable under radial perturbations.

\section{DISCUSSION}

The interpretation of a family of electrovacuum stationary Taub-NUT-type fields in terms of charged perfect fluid disks of finite extension was presented. The interpretation was done by means of an "inverse problem" approach used to obtain disk sources of known solutions of the Einstein or Einstein-Maxwell equations. The diagonalization of the energy-momentum tensor of the disks was facilitated by the fact that it was written as an upper right triangular matrix. We find that the inclusion of electromagnetic fields changes significatively the different material properties of the disks and so, for some values of the parameters, finite charged perfect fluid disks were obtained that are in agreement with all the energy conditions. However, the disks are not stable under radial perturbations. We also find that the disks have a barotropic equation of state.

\section{Acknowledgments}

The authors want to thank the finantial support from COLCIENCIAS, Colombia.
[1] D. Kramer, H. Stephani, E. Herlt, and M. McCallum, Exact Solutions of Einsteins's Field Equations (Cambridge University Press, Cambridge, England, 1980).

[2] W. A. Bonnor and A. Sackfield, Commun. Math. Phys. 8, 338 (1968).

[3] T. Morgan and L. Morgan, Phys. Rev. 183, 1097 (1969).

[4] L. Morgan and T. Morgan, Phys. Rev. D 2, 2756 (1970).

[5] A. Chamorro, R. Gregory, and J. M. Stewart, Proc. R.
Soc. London A413, 251 (1987).

[6] G. A. González and P. S. Letelier, Class. Quantum Grav. 16, 479 (1999).

[7] D. Vogt and P. S. Letelier, Phys. Rev. D. 68, 084010 (2003).

[8] D. Lynden-Bell and S. Pineault, Mon. Not. R. Astron. Soc. 185, 679 (1978).

[9] P.S. Letelier and S. R. Oliveira, J. Math. Phys. 28, 165 
(1987).

[10] J. P. S. Lemos, Class. Quantum Grav. 6, 1219 (1989).

[11] J. P. S. Lemos and P. S. Letelier, Class. Quantum Grav. 10, L75 (1993).

[12] J. Bičák, D. Lynden-Bell, and J. Katz, Phys. Rev. D 47, 4334 (1993).

[13] J. Bičák, D. Lynden-Bell, and C. Pichon, Mon. Not. R. Astron. Soc. 265, 126 (1993).

[14] J. Bičák and T. Ledvinka, Phys. Rev. Lett. 71, 1669 (1993).

[15] J. P. S. Lemos and P. S. Letelier, Phys. Rev D 49, 5135 (1994).

[16] J. P. S. Lemos and P. S. Letelier, Int. J. Mod. Phys. D 5, 53 (1996).

[17] C. Klein, Class. Quantum Grav. 14, 2267 (1997).

[18] G. A. González and P. S. Letelier, Phys. Rev. D 62, 064025 (2000).

[19] G.A. González and O. A. Espitia, Phys. Rev. D 68, 104028 (2003).

[20] T. Ledvinka, J. Bičák, and M. Žofka, in Procceeding of 8th Marcel-Grossmann Meeting in General Relativity, edited by T. Piran (World Scientific, Singapore, 1999)

[21] P. S. Letelier, Phys. Rev. D 60, 104042 (1999).

[22] J. Katz, J. Bičák, and D. Lynden-Bell, Class. Quantum Grav. 16, 4023 (1999).

[23] G. García R. and G. A. González, Phys. Rev. D 69, 124002 (2004).

[24] J. L. Synge, Relativity: The General Theory. (NorthHolland, Amsterdam, 1966).

[25] G. A. González and P. S. Letelier, Phys. Rev. D 69, 044013 (2004).

[26] D. Vogt and P. S. Letelier, Class. Quantum Grav. 21, 3369 (2004).

[27] F.J. Ernst, Phys. Rev. D 168, 1415 (1968).

[28] L.D. Landau and E.M. Lifshitz, Fluid Mechanics(Addisson-Wesley, Reading, MA, 1989).

[29] J.M. Bardeen, W.H Press, and S.A. Teukolsky, Astrophys. J. 178, 347 (1972).

[30] S. Chandrasekar, The Mathematical Theory of Black Holes. (Oxford University Press, 1992). 